\title{
Biocatalytic production of lactose ester catalysed by mica-based immobilised lipase
}

\begin{abstract}
Enzyme immobilisation technology is an effective means to improve sugar ester production through the employment of biocatalysts. In the present study, immobilisation of Candida rugosa (CRL) lipase onto amino-activated mica is performed via covalent bonding (namely Amino-CRL) and the cross-linking of lipases into nano-reactors through physical adsorption (namely NER-CRL). Free and immobilised lipases were tested for their esterification activities. Specific activities for Amino-CRL and NER-CRL increased by 2.4 and 2.6-fold, respectively, upon immobilisation. Extending this work, immobilised lipases have novel capabilities in the synthesis of sugar esters. The optimised conditions for sugar fatty acid ester syntheses are $48 \mathrm{~h}$ at $2: 1$ of molar ratio of lactose sugar to capric acid at $55{ }^{\circ} \mathrm{C}$. Furthermore, a high operational stability with half-lives of over 13 and 10 runs was achieved for NER-CRL and Amino-CRL, respectively, indicating the efficiency of the immobilisation process.
\end{abstract}

Keyword: Mica; Candida rugosa lipase; Covalent bonding; Cross-linking; Sugar esters 